\section{Morphological Detection of Plasma Membrane Changes During Apopto- sis Using Enhanced Green Fluorescent Protein}

BioTechniques 26:1064-1068 (June 1999)

Apoptosis, or programmed cell death, is one of the most widely studied areas of research today. The cellular alterations that occur during apoptosis have been well characterized. In fact, morphological changes to cells formed the basis of the original descriptions of apoptosis $(7,8)$. The hallmarks of apoptotic cell death include cell shrinkage, plasma membrane changes and nuclear fragmentation $(16,17)$. Changes in the plasma membrane have been widely observed during apoptosis, and these events are thought to be instructive for understanding how macrophages identify apoptotic cells. One of the earliest plasma membrane changes that occurs in response to apoptotic stimuli is the externalization of phosphatidylserine from the inner leaflet onto the outer membrane (3). The next series of events includes the formation of protrusions in the plasma membrane, which is usually referred to as membrane blebbing. Eventually the cell dissociates into membrane-bound particles that are referred to as apoptotic bodies. Finally, the apoptotic bodies are recognized and engulfed by macrophages (7). These changes are most often assessed using light or electron microscopy. In this study, we describe a novel noninvasive method to visualize plasma membrane changes that occur during apoptosis using fluorescence microscopy. We use a vector (pEGFP-F; CLONTECH Laboratories, Palo Alto, CA, USA) that targets enhanced green fluorescent protein $(2,5,11)$ to the plasma membrane $(1,6)$. Expression of EGFP-F results in a fluorescent green plasma membrane, allowing clear detection of morphological changes and providing the added benefit of marking transfected cells. We used EGFP-F to trace the progression of morphological changes that occur in the plasma membrane in response to apoptotic stimuli.

To visualize plasma-membrane morphological changes in response to apoptotic stimuli, we transfected $\mathrm{HeLa}$ cells with pEGFP-F ( $4 \mu \mathrm{g} / \mathrm{mL})$ using liposome-mediated transfection (19). EGFP-F contains the 20-amino acid farnesylation signal from c-Ha-Ras added to the $\mathrm{C}$ terminus of EGFP (6). The Ras farnesylation signal has been used in many studies to target heterologous proteins to the plasma membrane $(1,4)$. HeLa cells that were successfully transfected with EGFP-F were identified by their green fluorescent plasma membrane. After transfection, but before the addition of apoptotic stimuli, the HeLa cells appeared flat (Figure 1A). Transfection with EGFP-F did not have an effect on the morphological appearance of the cells (Figure 1A). The cells were induced to undergo apoptosis by addition of $1 \mu \mathrm{M}$ staurosporine (CLONTECH). Within $30 \mathrm{~min}$ after staurosporine addition, the cells began to appear round (Figure 1B). This change was readily detected due to the morphological changes emphasized by the fluorescent green membrane. Next, membrane blebbing was observed, and the borders of the bubble-like projections were accentuated due to EGFP fluorescence (Figure 1C). When the cell dissociated into apoptotic bodies (Figure 1, D and E), small membrane fragments were still visible. These morphological changes were clear and distinct due to the plasma membrane fluorescence from EGFP-F.

Standard methods use phase contrast or differential interference contrast (DIC/Nomarski) light microscopy to follow these changes. One advantage EGFP fluorescence offers over these methods is detection of the most subtle plasma membrane changes that would not be detected otherwise. However, electron microscopy is the best method to use for detailed study of apoptosis-induced ultrastructural changes. Another advantage of using EGFP fluorescence is resistance to photobleaching-it has been shown to be more resistant to photobleaching than fluorescein $(10,15)$. One limitation of the method we describe is that it involves transfection of cells with EGFP-F. In studies that require minimal cellular manipulation, an alternative method of detecting apoptotic cells should be used. In this context, plasma membrane changes can be examined using less invasive methods such as staining the cells with annexin V-fluorescein isothiocyanate (FITC) $(12-14,18)$. The annexin V moiety binds to the phosphatidylserine exposed on apoptotic cells, whereas the FITC conjugate allows fluorescent detection of these cells.

The ability to track plasma membrane changes offers many advantages. EGFP-F can be used in co-transfection experiments to determine the ability of transfected genes to inhibit or induce apoptosis. In previous studies, cytoplasmic EGFP had been co-transfected with known apoptosis-inducing genes, tumor necrosis factor-R (TNF-R) and Fas (9), to identify positively transfected cells. It was reported that the cotransfection of EGFP had no negative cellular effects, while it provided a convenient method to identify transfected cells (9). In our experience, we found 
that EGFP-F provided the additional benefit of marking the plasma membrane, making it suitable for studies involving plasma-membrane morphology. Figure 1F illustrates this application. The transfected cells within the well are clearly marked green. Induction of apoptotsis in response to staurosporine was detected by the clear delineation of membrane blebbing. We used this method to determine the ability of a newly isolated gene to induce apoptosis (unpublished results). The ef- fect of the gene of interest was easily detected by following the fluorescent patterns of EGFP-F expression (data not shown). Co-transfection with EGFP-F had the added benefit of insurserved was due to transfection of the unknown gene, because transfected cells were labeled green. This application could be useful for apoptosis studies, along with any study that involves monitoring changes in plasma-membrane morphology. ing that the cellular phenotype ob-
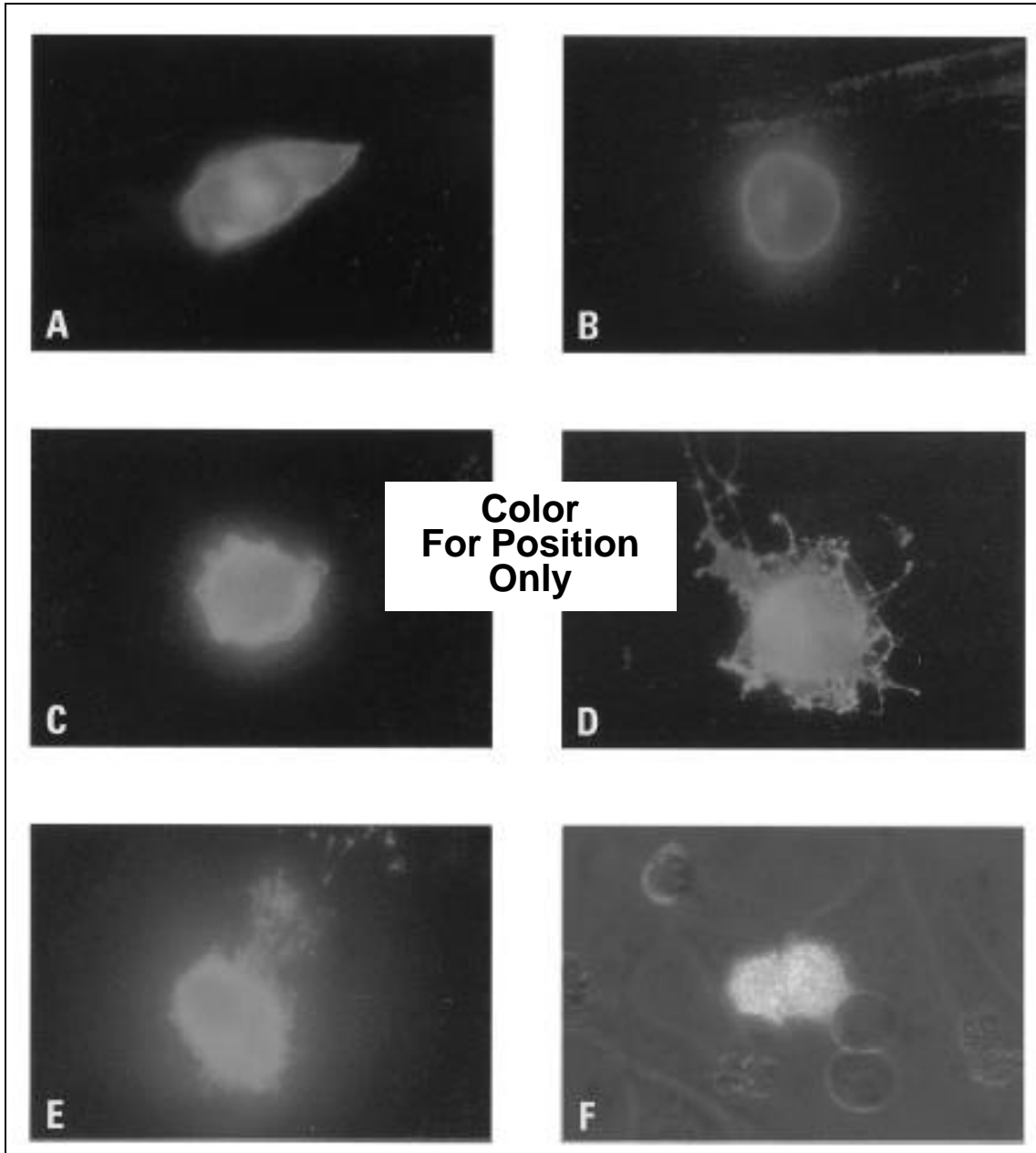

Figure 1. Analysis of cells transfected with pEGFP-F. $1 \times 10^{5} \mathrm{HeLa}$ cells were plated in Falcon ${ }^{\circledR} 2$ well chamber slides (Becton Dickinson Labware, Bedford, MA, USA). The cells were transfected with 4 $\mu \mathrm{g} / \mathrm{mL}$ pEGFP-F using CLONfectin ${ }^{\mathrm{TM}}$ Transfection Reagent (CLONTECH) according to the manufacturer's protocol. Cells were examined for expression of the EGFP-F transgene $24 \mathrm{~h}$ after transfection. (A) Transfected cells display a green fluorescent plasma membrane, the cell is elongated and attached. (B) Apoptosis was induced by the addition of $1 \mu \mathrm{M}$ staurosporine. The cell starts to appear round, and is less attached to the plate surface. (C) The characteristic apoptotic membrane blebbing is clearly visible, and EGFP-F fluorescence outlines various bubble-like projections from the plasma membrane. (D) Membrane convolutions include extensive surface projections, which start to form membrane-bound cytoplasmic fragments. (E) Apoptotic bodies form from membrane-bound cellular fragments. (F) Positively transfected HeLa cells with EGFP-F are clearly distinguishable from nontransfected neighbors. The effect of $1 \mu \mathrm{M}$ staurosporine is clearly visible in EGFP-F transfected cells. Membrane blebbing is clear and detectable.

\section{REFERENCES}

1.Aronheim, A., D. Engelberg, N. Li, N. alAlawi, J. Schlessinger and M. Karin. 1994. Membrane targeting of the nucleotide exchange factor Sos is sufficient for activating the Ras signaling pathway. Cell 78:949-961.

2.Chalfie, M., Y. Tu, G. Euskirchen, W.W. Ward and D.C. Prasher. 1994. Green fluorescent protein as a marker for gene expression. Science 263:802-805.

3.Fadok, V.A., D.R. Voelker, P.A. Campbell, J.J. Cohen, D.L. Bratton and P.M. Henson. 1992. Exposure of phosphatidylserine on the surface of apoptotic lymphocytes triggers specific recognition and removal by macrophages. J. Immunol. 148:2207-2216.

4.Hancock, J.F., K. Cadwallader, H. Paterson and C.J. Marshall. 1991. A CAAX or a CAAL motif and a second signal are sufficient for plasma membrane targeting of ras proteins. EMBO J. 10:4033-4039.

5.Inouye, S. and F.I. Tsuji. 1994. Evidence for redox forms of the Aequorea victoria green fluorescent protein. FEBS Lett. 351:211-214.

6.Jiang, W. and T. Hunter. 1998. Analysis of cell-cycle profiles in transfected cells using a membrane- targeted GFP. BioTechniques 24:349-354.

7.Kerr, J.F. 1971. Shrinkage necrosis: a distinct mode of cellular death. J. Pathol. 105:13-20.

8.Kerr, J.F., A.H. Wyllie and A.R. Currie. 1972. Apoptosis: a basic biological phenomenon with wide-ranging implications in tissue kinetics. Br. J. Cancer 26:239-257.

9.Li, Y. and M.S. Horwitz. 1997. Use of green fluorescent protein in studies of apoptosis of transfected cells. BioTechniques 23:10261029.

10.Niswender, K.D., S.M. Blackman, L. Rohde, M.A. Magnuson and D.W. Piston. 1995. Quantitative imaging of green fluorescent protein in cultured cells: comparison of microscopic techniques, use in fusion proteins and detection limits. J. Microsc. 180:109-116.

11.Prasher, D.C., V.K. Eckenrode, W.W. Ward, F.G. Prendergast and M.J. Cormier. 1992. Primary structure of the Aequorea victoria green-fluorescent protein. Gene 111:229-233.

12.Tait, J.F., D. Gibson and K. Fujikawa. 1989. Phospholipid binding properties of human placental anticoagulant protein-I, a member of the lipocortin family. J. Biol. Chem. 264:79447949

13.van Engeland, M., L.J. Nieland, F.C. Ramaekers, B. Schutte and C.P. Reutelingsperger. 1998. Annexin V-affinity assay: a review on an apoptosis detection system based on phosphatidylserine exposure. Cytometry 31:1-9.

14.van Engeland, M., F.C. Ramaekers, B. Schutte and C.P. Reutelingsperger. 1996. A novel assay to measure loss of plasma membrane asymmetry during apoptosis of adherent cells in culture. Cytometry 24:131-139.

15.Wang, S. and T. Hazelrigg. 1994. Implications for bed mRNA localization from spatial distribution of exu protein in Drosophila oogenesis. Nature 369:400-403.

16.Wyllie, A.H. 1980. Glucocorticoid-induced thymocyte apoptosis is associated with endogenous endonuclease activation. Nature 
284:555-556.

17.Wyllie, A.H., J.F. Kerr and A.R. Currie. 1980. Cell death: the significance of apoptosis. Int. Rev. Cytol. 68:251-306.

18.Zhang, G., V. Gurtu, S.R. Kain and G. Yan. 1997. Early detection of apoptosis using a fluorescent conjugate of annexin V. BioTechniques 23:525-531.

19.Zhang, G., V. Gurtu, T.H. Smith, P. Nelson and S.R. Kain. 1997. A cationic lipid for rapid and efficient delivery of plasmid DNA into mammalian cells. Biochem. Biophys. Res. Commun. 236:126-129.

Address correspondence to Dr. Eve Shinbrot, CLONTECH Laboratories, 1020 East Meadow Circle, Palo Alto, CA 94303, USA. Internet: exshinbrot@clontech.com

Received 16 December 1998; accepted 23 February 1999.

Eve Shinbrot, Collin M. Spencer and Steven R. Kain CLONTECH Laboratories

Palo Alto, CA, USA

\section{Extending the Useful Life Span of DNA Probes for Fluorescence In Situ Hybridization}

BioTechniques 26:1068-1072 (June 1999)

Whole-chromosome painting probes for fluorescence in situ hybridization (FISH) are comprised of thousands of unique sequences, each of which is represented by numerous copies of the original. These probes are frequently made by using degenerate oligonucleotide-primed polymerase chain reaction (DOP-PCR) to amplify DNA obtained by microdissection or flow sorting $(2,4)$. Once chromosomes have been obtained by one of these methods, they are subjected to 30 or 40 cycles of PCR. This product becomes the original stock solution, and an aliquot is then labeled with a reporter molecule (e.g., biotin or rhodamine) and used as a painting probe for FISH. When this probe is gone, more is made from the unlabeled PCR products. To avoid the necessity of flow sorting or microdissecting again, an aliquot of the original stock is re-amplified without a label, providing a new stock of unlabeled DNA from which probe can be made.

Such probes have a finite useful life span, which is partially determined by the number of PCR cycles through which the probe has been taken. Each time the stock DNA is replenished by PCR, the resulting product tends to have less complexity than the precursor. A complex probe will contain enough specific sequences to paint a chromosome evenly; stochastic losses of individual DNA sequences tend to result in a probe that yields uneven labeling and hence shows a banded pattern. The primary reason for the loss of 